\title{
Generalized Analytic Difference Sequence Spaces Defined By Musielak-Orlicz Function
}

\author{
Zakawat U. Siddiqui and Ado Balili \\ Department of Mathematics and Statistics, University of Maiduguri, Borno State, Nigeria
}

Abstract: In this paper, the generalized analytic difference sequence space defined by Musielak-Orlicz function, are introduced, and some of their algebraic and topological properties are explored. Few inclusion relations involving the introduced spaces are also discussed.

Keywords: Analytic Sequences, Difference Sequence Space, Entire Sequences, Musielak- Orlicz Function,

\section{Introduction}

A complex sequence, whose $k^{\text {th }}$ term is denoted by $\left(x_{k}\right)$.A sequence $\mathrm{x}=\left(x_{k}\right)$ is said to be analytic, if $\sup _{k}\left|x_{k}\right|^{\frac{1}{k}}<\infty$. The vector space of all analytic sequences will be denoted by $\Lambda$. A sequence is entire sequence, if $\lim _{k}\left|x_{k}\right|^{\frac{1}{k}}=0$. The vector space of all entire sequences will be denoted by $\Gamma$.

The notion of difference sequence space was introduced by Kizmaz [1], who studied the difference sequence spaces $\ell_{\infty}(\Delta), \mathrm{c}(\Delta)$ and $c_{0}(\Delta)$. Kizmaz defined the following Difference Sequence Spaces:

$$
\mathrm{Z}(\Delta)=\left\{x=\left(x_{k}\right): \Delta_{\mathrm{x}} \in \mathrm{Z}\right\} \text { where } \Delta_{\mathrm{x}}=\left(\Delta_{\mathrm{x}}\right)_{\mathrm{k}=1}^{\infty}=\left(\mathrm{x}_{\mathrm{k}}-\mathrm{x}_{\mathrm{k}+1}\right)_{\mathrm{k}=1}^{\infty} \text {. }
$$

Here $\mathrm{Z}$ stands for one of the spaces $c_{0}, c$ and $\ell_{\infty}$.

The notion was further generalized by Et and Colak [2] by introducing the spaces $c\left(\Delta^{m}\right), c_{0}\left(\Delta^{m}\right)$ and $\ell_{\infty}\left(\Delta^{m}\right)$

Let $m, v$ be non-negative integers, then for $z=\left\{\ell_{\infty}, c, c_{0}\right\}$, we have sequence spaces

$$
\mathrm{Z}\left(\Delta_{v}^{m}\right)=\left\{x=\left(x_{k}\right) \in \omega:\left(\Delta_{v}^{m} x_{k}\right) \in Z\right\} \quad \text { (see Raji et al [3]) }
$$

where $\Delta_{v}^{m} x=\left(\Delta_{v}^{m} x_{k}\right)=\left(\Delta_{v}^{m-1} x_{k}-\Delta_{v}^{m-1} x_{k+1}\right)$ and $\Delta_{v}^{0} x_{k}=x_{k}, \forall k \in \mathbb{N}$, which is equivalent to the following binomial expansion

$$
\Delta_{v}^{m} x_{k}=\sum_{i=0}^{m}(-1)^{i}\left(\begin{array}{c}
m \\
i
\end{array}\right) x_{k+i v}
$$

Taking $v=1$, we have the spaces which were studied by Et and Colak [2].

Taking $m=v=1$, we get the spaces which were introduced and studied by Kizmaz [1].

An Orlicz function $\mathrm{M}:[0, \infty) \rightarrow[0, \infty)$ is a continuous, non decreasing and convex function such that $M(0)$ $=0, M(x)>0$, for all $x>0$ and $M(x) \rightarrow 0$ as $x \rightarrow \infty$.

Lindenstrauss and Tzafriri [4] used the idea of Orlicz function to define the following sequence space:

$$
\ell_{M}=\left\{x \in \omega: \sum_{k=1}^{\infty} M\left(\frac{\left|x_{k}\right|}{\rho}\right)<\infty\right\},
$$

where $\omega=$ \{all complex sequences $\}$, which is called an Orlicz sequence space. Also $\ell_{M}$ is a Banach space with norm

$$
\|x\|=\operatorname{infin}\left\{\rho>0: \sum_{k=1}^{\infty} M\left(\frac{\left|x_{k}\right|}{\rho}\right) \leq 1\right\}
$$

It was proved that every Orlicz sequence space $\ell_{M}$ contains a subspace isomorphic to $\ell_{p}(1 \leq p<\infty)$

\section{Definitions And Preliminaries}

Definition 2.1: Let $M_{k}$ be an Orlicz function. The space consisting of all these sequences $\mathrm{x}$ in $\omega$, such that $\sup _{k}\left(M_{k}\left(\frac{\left|x_{k}\right|^{\frac{1}{k}}}{\rho}\right)\right)<\infty$ for some arbitrary fixed $\rho>0$ is denoted by $\Lambda_{M}$ and is known as the space of analytic sequence defined by a sequence of Orlicz function.

Definition 2.2 (see (Musielak [5]): A sequence space $\mathrm{E}$ is said to be solid or normal if $\left(\alpha_{k} x_{k}\right) \in E$, where $\left(x_{k}\right) \in E$ and for all sequence of scalars $\left(\alpha_{k}\right)$ with $\left|\alpha_{k}\right| \leq 1$.

Definition 2.3: Let $\mathrm{V}$ be a vector space over scalar field $\mathrm{K}$. A seminorm $\mathrm{v}$ on $\mathrm{V}$ is a real valued function on $\mathrm{V}$ so that

1. $\quad \mathrm{v}(\mathrm{x}) \geq 0$, for all $x \in V$

2. $\mathrm{v}(\alpha x)=|\alpha| v(x)$, for all $\alpha \in k, x \in V$

3. $\mathrm{v}(\mathrm{x}+\mathrm{y}) \leq v(x)+v(y)$, for all $x, y \in V$

Definition 2.4 (Maddox [6]): Let $\mathrm{X}$ be a linear metric space. A function $p: X \rightarrow R$ is called paranorm, if

1. $p(x) \geq 0, \forall x \in X$,

2. $p(-x)=p(x), \forall x \in X$,

3. $p(x+y) \leq p(x)+p(y), \forall x, y \in X$. 
4. If $\left(\lambda_{n}\right)$ is a sequence of scalars with $\lambda_{n} \rightarrow \lambda$ as $n \rightarrow \infty$ and $\left(x_{n}\right)$ is a sequence of vectors with $p\left(x_{n}-\right.$ $x \rightarrow 0$ as $n \rightarrow \infty$, then $p \lambda n x n-\lambda x \rightarrow 0$ as $n \rightarrow \infty$.

Remark 2.1: The following inequality will be used throughout the paper.

Let $p=\left(p_{k}\right)$ be a sequence of positive real numbers with $0 \leq p_{k} \leq \sup \left(p_{k}\right)=G, K=\max \left(1,2^{G-1}\right)$, then

$$
\left|a_{k}+b_{k}\right|^{p_{k}} \leq K\left\{\left|a_{k}\right|^{p_{k}}+\left|b_{k}\right|^{p_{k}}\right\}
$$

where $a_{k}, b_{k} \in \mathbb{C}$. Also $|a|^{p_{k}} \leq \max \left(1,|a|^{G}\right)$ for all $a \in \mathbb{C}$.

Definition 2.5 (Musielak [5]): Musielak-Orlicz function is defined to be a sequence of Orlicz functions.

Definition 2.6: Let $\mathcal{M}=\left(M_{k}\right)$ be a sequence of Orlicz functions, $\mathrm{X}$ be locally convex Hausdorff topological linear space whose topology is determined by a set of continuous seminorms q. The symbols $\Lambda(X)$ and $\Gamma(X)$ denote the space of all analytic and entire sequences, respectively defined over X.

Now we define a new sequence space:

$\Lambda_{\mathcal{M}}\left(\Delta_{v}^{m}, p, q, s\right)=\left\{x \in \Lambda(X): \sup _{n} \frac{1}{n} \sum_{k=1}^{n} k^{-s}\left[M_{k}\left(q\left(\frac{\left|\Delta_{v}^{m} x_{k}\right|^{\frac{1}{k}}}{\rho}\right)\right)\right]^{p_{k}}<\infty\right.$, for some $\left.\rho>0\right\}$

Remarks 2.2: We get the following analytic sequence spaces from the above space by giving particular values to $\mathrm{p}$ and $\mathrm{s}$.

Taking $p_{k}=1$, for all $\mathrm{n} \in \mathbb{N}$,we have

$\Lambda_{\mathcal{M}}\left(\Delta_{v}^{m}, q, s\right)=\left\{x \in \Lambda(X): \sup _{n} \frac{1}{n} \sum_{k=1}^{n} k^{-s}\left[M_{k}\left(q\left(\frac{\Delta_{v}^{m} x_{k} k^{\frac{1}{k}}}{\rho}\right)\right)\right]<\infty\right.$, for some $\left.\rho>0\right\}$ (Abbas and Kamel [7])

If we take $s=0$, we have

$\Lambda_{\mathcal{M}}\left(\Delta_{v}^{m}, p, q\right)=\left\{x \in \Lambda(X): \sup _{n} \frac{1}{n} \sum_{k=1}^{n}\left[M_{k}\left(q\left(\frac{\left|\Delta_{v}^{m} x_{k}\right|^{\frac{1}{k}}}{\rho}\right)\right)\right]^{p_{k}}<\infty\right.$, for some $\left.\rho>0\right\} \quad$ (Raj et al [3] )

If we take $\mathrm{s}=0, \mathrm{~m}=\mathrm{v}=1$, we get

$\Lambda_{\mathcal{M}}(\Delta, p, q)=,\left\{x \in \Lambda(X): \sup _{n} \frac{1}{n} \sum_{k=1}^{n}\left[M_{k}\left(q\left(\frac{\left|\Delta x_{k}\right|^{\frac{1}{k}}}{\rho}\right)\right)\right]^{p_{k}}<\infty\right.$, for some $\left.\rho>0\right\}$

(Lindenstrauss and Tzafriri [4])

\section{Main Results}

The following results are obtained in this work.

Theorem 3.1: Let $\mathrm{M}=\left(M_{k}\right)$ be any Musielak Orlicz function, and $p=\left(p_{k}\right)$ a sequence of strictly positive real numbers, then $\Lambda_{\mathcal{M}}\left(\Delta_{v}^{m}, p, q, s\right)$ is a linear space over the set of complex numbers $\mathbb{C}$.

Proof. Let $x=\left(x_{k}\right), y=\left(y_{k}\right) \in \Lambda_{\mathcal{M}}\left(\Delta_{v}^{m}, p, q, s\right)$ and $\alpha, \beta \in \mathbb{C}$, then we have

$$
\begin{aligned}
& \sup _{n} \frac{1}{n} \sum_{k=1}^{n} k^{-s}\left[M_{k}\left(q\left(\frac{\left.\Delta_{v}^{m} x_{k}\right|^{\frac{1}{k}}}{\rho_{1}}\right)\right)\right]^{p_{k}}<\infty \text {, for some } \rho_{1}>0 \\
& \sup _{n} \frac{1}{n} \sum_{k=1}^{n} k^{-s}\left[M_{k}\left(q\left(\frac{\left.\Delta_{v}^{m} y_{k}\right|^{\frac{1}{k}}}{\rho_{2}}\right)\right)\right]^{p_{k}}<\infty \text {, for some } \rho_{2}>0
\end{aligned}
$$

Since $M=\left(M_{k}\right)$ is a non decreasing modulus function, q seminorm and $\Delta_{v}^{m}$ is linear, then

$$
\sup _{n} \frac{1}{n} \sum_{k=1}^{n} k^{-s}\left[M_{k}\left(q\left(\frac{\left.\Delta_{v}^{m}\left(\alpha x_{k}+\beta y_{k}\right)\right|^{\frac{1}{k}}}{\rho_{3}}\right)\right)\right]^{p_{k}}<\infty \text {, for some } \rho_{3}>0
$$

where $\left.\rho_{3}=\max \left|\frac{1}{t} \alpha\right|^{\frac{1}{k}} \rho_{1},|\beta|^{\frac{1}{k}} \rho_{2}\right\}$.

Now,

$$
\begin{aligned}
& \sup _{n} \frac{1}{n} \sum_{k=1}^{n} k^{-s}\left[M_{k}\left(q\left(\frac{\left|\Delta_{v}^{m}\left(\alpha x_{k}+\beta y_{k}\right)\right|^{\frac{1}{k}}}{\rho_{3}}\right)\right)\right]^{p_{k}} \\
& \quad \leq \sup _{n} \frac{1}{n} \sum_{k=1}^{n} k^{-s}\left[M_{k}\left(q\left(\frac{|\alpha|^{\frac{1}{k}}\left|\Delta_{v}^{m} x_{k}\right|^{\frac{1}{k}}}{\rho_{3}}+\frac{|\beta|^{\frac{1}{k}}\left|\Delta_{v}^{m} y_{k}\right|^{\frac{1}{k}}}{\rho_{3}}\right)\right)\right]^{p_{k}} \\
& \leq \operatorname{Kup}_{n} \frac{1}{n} \sum_{k=1}^{n} k^{-s}\left[M_{k}\left(q\left(\frac{\left|\Delta_{v}^{m} x_{k}\right|^{\frac{1}{k}}}{\rho_{1}}+\frac{\left|\Delta_{v}^{m} y_{k}\right|^{\frac{1}{k}}}{\rho_{2}}\right)\right)\right]^{p_{k}}
\end{aligned}
$$




$$
\begin{gathered}
\leq K \sup _{n} \frac{1}{n} \sum_{k=1}^{n} k^{-s}\left[M_{k}\left(q\left(\frac{\left|\Delta_{v}^{m} x_{k}\right|^{\frac{1}{k}}}{\rho_{1}}\right)\right)+M_{k}\left(q\left(\frac{\left|\Delta_{v}^{m} y_{k}\right|^{\frac{1}{k}}}{\rho_{2}}\right)\right)\right]^{P_{K}} \\
\leq \operatorname{Kup}_{n} \frac{1}{n} \sum_{k=1}^{n} k^{-s}\left[M_{k}\left(q\left(\frac{\left|\Delta_{v}^{m} x_{k}\right|^{\frac{1}{k}}}{\rho_{1}}\right)\right)+K \sup _{n} \frac{1}{n} \sum_{k=1}^{n} k^{-s}\left[M_{k}\left(q\left(\frac{\left|\Delta_{v}^{m} y_{k}\right|^{\frac{1}{k}}}{\rho_{2}}\right)\right)\right]^{P_{K}}<\infty\right.
\end{gathered}
$$

This proves that $\Lambda_{\mathcal{M}}\left(\Delta_{v}^{m}, p, q, s\right)$ is a linear space.

Theorem 3.2: Let $\mathcal{M}^{\prime}=\left(M_{k}^{\prime}\right)$ and $\mathcal{M}^{\prime \prime}=\left(M_{k}^{\prime \prime}\right)$ be Musielak- Orlicz functions. Then

$$
\Lambda_{\mathcal{M}^{\prime}}\left(\Delta_{v}^{m}, p, q, s\right) \cap \Lambda_{\mathcal{M}^{\prime \prime}}\left(\Delta_{v}^{m}, p, q, s\right) \subseteq \Lambda_{\mathcal{M}^{\prime}+\mathcal{M}^{\prime \prime}}\left(\Delta_{v}^{m}, p, q, s\right) .
$$

Proof. Let $x \in \Lambda_{\mathcal{M}^{\prime}}\left(\Delta_{v}^{m}, p, q, s\right) \cap \Lambda_{\mathcal{M}^{\prime \prime}}\left(\Delta_{v}^{m}, p, q, s\right)$.Then there exist $\rho_{1}$ and $\rho_{2}$ such that

$$
\begin{aligned}
& \sup _{n} \frac{1}{n} \sum_{k=1}^{n} k^{-s}\left[M_{k}^{\prime}\left(q\left(\frac{\Delta \frac{\Delta x_{v}}{\rho_{1}} \frac{1}{k}}{k}\right)\right)\right]^{p_{k}}<\infty \text {, for some } \rho_{1}>0 \\
& \sup _{n} \frac{1}{n} \sum_{k=1}^{n} k^{-s}\left[M_{k}^{\prime \prime}\left(q\left(\frac{\left|\Delta_{v}^{m} x_{k}\right| \frac{1}{k}}{\rho_{2}}\right)\right)\right]^{p_{k}}<\infty \text {, for some } \rho_{2}>0
\end{aligned}
$$

Let $\rho=\min \left(\frac{1}{\rho_{1}}, \frac{1}{\rho_{2}}\right)$. Then we have

$$
\begin{aligned}
\sup _{n} \frac{1}{n} \sum_{k=1}^{n} k^{-s}\left[\left(M_{k}^{\prime}+M_{k}^{\prime \prime}\right)\left(q\left(\frac{\left|\Delta_{v}^{m} x_{k}\right| \frac{1}{k}}{\rho}\right)\right)\right]^{p_{k}} & \leq \operatorname{Ksup}_{n} \frac{1}{n} \sum_{k=1}^{n} k^{-s}\left[M_{k}^{\prime}\left(q\left(\frac{\left|\Delta_{v}^{m} x_{k}\right| \frac{1}{k}}{\rho_{1}}\right)\right)\right]^{p_{k}} \\
& +\operatorname{Ksup}_{n} \frac{1}{n} \sum_{k=1}^{n} k^{-s}\left[M_{k}^{\prime \prime}\left(q\left(\frac{\left|\Delta_{v}^{m} x_{k}\right| \frac{1}{k}}{\rho_{2}}\right)\right)\right]^{p_{k}}<\infty
\end{aligned}
$$

by (3.21) and (3.21). Then

$\sup _{n} \frac{1}{n} \sum_{k=1}^{n} k^{-s}\left[\left(M_{k}^{\prime}+M_{k}^{\prime \prime}\right)\left(q\left(\frac{\left.\Delta \Delta_{v}^{m} x_{k}\right|^{\frac{1}{k}}}{\rho}\right)\right)\right]^{p_{k}}<\infty$ for some $\rho>0$

Therefore, $x \in \Lambda_{\mathcal{M}^{\prime}+\mathcal{M}^{\prime \prime}}\left(\Delta_{v}^{m}, p, q, s\right)$.

Theorem 3.3: The sequence space $\Lambda_{\mathcal{M}}\left(\Delta_{v}^{m}, p, q, s\right)$ is solid.

Proof. Let $x=\left(x_{k}\right) \in \Lambda_{\mathcal{M}}\left(\Delta_{v}^{m}, p, q, s\right)$, then

$$
\sup _{n} \frac{1}{n} \sum_{k=1}^{n} k^{-s}\left[M_{k}\left(q\left(\frac{\Delta_{v}^{m} x_{k} \frac{1}{\rho}}{\rho}\right)\right)\right]^{p_{k}}<\infty .
$$

Let $\left(\alpha_{k}\right)$ be a sequence of scalars such that $\left|\alpha_{k}\right| \leq 1, \forall k \in \mathbb{N}$, then we have

Hence, $\left(\alpha_{k} x_{k}\right) \in \Lambda_{\mathcal{M}}\left(\Delta_{v}^{m}, p, q, s\right)$.

$$
\begin{gathered}
\sup _{n} \frac{1}{n} \sum_{k=1}^{n} k^{-s}\left[M_{k}\left(q\left(\frac{\left|\alpha_{k} \Delta_{v}^{m} x_{k}\right|^{\frac{1}{k}}}{\rho}\right)\right)\right]^{p_{k}} \leq \sup _{n} \frac{1}{n} \sum_{k=1}^{n} k^{-s}\left[M_{k}\left(q\left(\frac{\left.\left|\alpha_{k} \frac{1}{\mid}\right| \Delta_{v}^{m} x_{k}\right|^{\frac{1}{k}}}{\rho}\right)\right)\right]^{p_{k}} \\
\leq \sup _{n} \frac{1}{n} \sum_{k=1}^{n} k^{-s}\left[M_{k}\left(q\left(\frac{\left|\Delta_{v}^{m} x_{k}\right|^{\frac{1}{k}}}{\rho}\right)\right)\right]^{p_{k}}<\infty
\end{gathered}
$$

Theorem 3.4: Suppose $\sup _{n} \frac{1}{n} \sum_{k=1}^{n} k^{-s}\left[M_{k}\left(q\left(\frac{\mid \Delta_{v}^{m} x_{k} \frac{1}{\rho}}{\rho}\right)\right)\right]^{p_{k}} \leq\left|x_{k}\right|^{\frac{1}{k}}$, then $\Lambda \subset \Lambda_{\mathcal{M}}\left(\Delta_{v}^{m}, p, q, s\right)$.

Proof. Let $x \in \Lambda$. Then we have,

$$
\sup \left|x_{k}\right|^{\frac{1}{k}}<\infty .
$$

But

$$
\sup _{n} \frac{1}{n} \sum_{k=1}^{n} k^{-S}\left[M_{k}\left(q\left(\frac{\left|v_{v}^{m} x_{k}\right|^{\frac{1}{k}}}{\rho}\right)\right)\right]^{p_{k}} \leq \sup \left|x_{k}\right|^{\frac{1}{k}}
$$

by our assumption. It implies that

$$
\sup _{n} \frac{1}{n} \sum_{k=1}^{n} k^{-s}\left[M_{k}\left(q\left(\frac{\Delta_{v}^{m} x_{k} k^{\frac{1}{k}}}{\rho}\right)\right)\right]^{p_{k}} \leq \infty
$$

by (3.41). Then $x \in \Lambda_{\mathcal{M}}\left(\Delta_{v}^{m}, p, q, s\right)$ and $\Lambda \subset \Lambda_{\mathcal{M}}\left(\Delta_{v}^{m}, p, q, s\right)$. 
Theorem 3.5: Let $0 \leq p_{k} \leq r_{k}$ and $\left\{\frac{r_{k}}{p_{k}}\right\}$ be bounded. Then $\Lambda_{\mathcal{M}}\left(\Delta_{v}^{m}, r, q, s\right) \subset \Lambda_{\mathcal{M}}\left(\Delta_{v}^{m}, p, q, s\right)$.

Proof. Let $x \in \Lambda_{\mathcal{M}}\left(\Delta_{v}^{m}, r, q, s\right)$, then

$$
\sup _{n} \frac{1}{n} \sum_{k=1}^{n} k^{-S}\left[M_{k}\left(q\left(\frac{\left.\Delta_{v}^{m} x_{k}\right|^{\frac{1}{k}}}{\rho}\right)\right)\right]^{r_{k}}<\infty .
$$

Let

$$
t_{k}=\sup _{n} \frac{1}{n} \sum_{k=1}^{n} k^{-s}\left[M_{k}\left(q\left(\frac{\left|\Delta_{v}^{m} x_{k}\right|^{\frac{1}{k}}}{\rho}\right)\right)\right]^{q_{k}}
$$

and $\lambda_{k}=\frac{p_{k}}{r_{k}}$. Since $p_{k} \leq r_{k}$, we have $0 \leq \lambda_{k} \leq 1$.

Take $0<\lambda<\lambda_{k}$. Define

and

$$
u_{k}= \begin{cases}t_{k}, & \text { if } t_{k} \geq 1 \\ 0, & \text { if } t_{k}<1\end{cases}
$$

$$
v_{k}= \begin{cases}0, & \text { if } t_{k} \geq 1 \\ t_{k}, & \text { if } t_{k}<1\end{cases}
$$

$t_{k}=u_{k}+v_{k}, \quad t_{k}^{\lambda_{k}}=u_{k}^{\lambda_{k}}+v_{k}^{\lambda_{k}}$. It follows that $u_{k}^{\lambda_{k}} \leq u_{k} \leq t_{k}, v_{k}^{\lambda_{k}} \leq v_{k}{ }^{\lambda}$. Since $t_{k}^{\lambda_{k}}=u_{k}^{\lambda_{k}}+$ $v_{k}^{\lambda_{k}}$, then $t_{k}^{\lambda_{k}} \leq t_{k}+v_{k}^{\lambda}$. Thus

$\sup _{n} \frac{1}{n} \sum_{k=1}^{n} k^{-s}\left[M_{k}\left(q\left(\frac{\left|\Delta_{k}^{m} x_{k}\right|^{\frac{1}{k}}}{\rho}\right)\right)^{r_{k}}\right]^{\lambda_{k}} \leq \sup _{n} \frac{1}{n} \sum_{k=1}^{n} k^{-s}\left[M_{k}\left(q\left(\frac{\left|\Delta_{k}^{m} x_{k}\right|^{\frac{1}{k}}}{\rho}\right)\right)\right]^{r_{k}}$

$\Rightarrow \sup _{n} \frac{1}{n} \sum_{k=1}^{n} k^{-s}\left[M_{k}\left(q \frac{\left|\Delta_{k}^{m} x_{k}\right|^{\frac{1}{k}}}{\rho}\right)^{r_{k}}\right]^{\frac{p_{k}}{r_{k}}} \leq \sup _{n} \frac{1}{n} \sum_{k=1}^{n} k^{-s}\left[M_{k}\left(q\left(\frac{\left|\Delta_{k}^{m} x_{k}\right|^{\frac{1}{k}}}{\rho}\right)\right)\right]^{r_{k}}$

$\Rightarrow \sup _{n} \frac{1}{n} \sum_{k=1}^{n} k^{-s}\left[M_{k}\left(q\left(\frac{\left|\Delta_{k}^{m} x_{k}\right|^{\frac{1}{k}}}{\rho}\right)\right)^{r_{k}}\right]^{p_{k}} \leq \sup _{n} \frac{1}{n} \sum_{k=1}^{n} k^{-s}\left[M_{k}\left(q\left(\frac{\left|\Delta_{k}^{m} x_{k}\right|^{\frac{1}{k}}}{\rho}\right)\right)\right]^{r_{k}}$

But, $\sup _{n} \frac{1}{n} \sum_{k=1}^{n} k^{-s}\left[M_{k}\left(q\left(\frac{\left|\Delta_{k}^{m} x_{k}\right|^{\frac{1}{k}}}{\rho}\right)\right)\right]^{r_{k}}<\infty$.

Therefore, $\sup _{n} \frac{1}{n} \sum_{k=1}^{n} k^{-s}\left[M_{k}\left(q\left(\frac{\left|\Delta_{k}^{m} x_{k}\right|^{\frac{1}{k}}}{\rho}\right)\right)^{r_{k}}\right]^{p_{k}}<\infty$.

Hence $x \in \Lambda_{\mathcal{M}}\left(\Delta_{v}^{m}, p, q, s\right)$. From (3.51), we get

$$
\Lambda_{\mathcal{M}}\left(\Delta_{v}^{m}, r, q, s\right) \subset \Lambda_{\mathcal{M}}\left(\Delta_{v}^{m}, p, q, s\right) .
$$

Theorem 3.6 (i) Let $0<$ inf $p_{k} \leq p_{k} \leq 1$. Then, $\Lambda_{\mathcal{M}}\left(\Delta_{v}^{m}, p, q, s\right) \subset \Lambda_{\mathcal{M}}\left(\Delta_{v}^{m}, q, s\right)$

(ii) Let $1 \leq p_{k} \leq \sup p_{k}<\infty$. Then $\Lambda_{\mathcal{M}}\left(\Delta_{v}^{m}, q, s\right) \subset \Lambda_{\mathcal{M}}\left(\Delta_{v}^{m}, p, q, s\right)$

Proof. (i) Let $\left.x \in \Lambda_{\mathcal{M} \mathcal{A}} \Delta_{v}^{m}, p, q, s\right)$. Then

$$
\sup _{n} \frac{1}{n} \sum_{k=1}^{n} k^{-s}\left[M_{k}\left(q\left(\frac{\left|\Delta_{v}^{m} x_{k}\right|^{\frac{1}{k}}}{\rho}\right)\right)\right]^{p_{k}}<\infty
$$

Since $0<\inf p_{k} \leq p_{k} \leq 1$,

$\sup _{n} \frac{1}{n} \sum_{k=1}^{n} k^{-s}\left[M_{k}\left(q\left(\frac{\left|\Delta_{v}^{m} x_{k}\right|^{\frac{1}{k}}}{\rho}\right)\right)\right] \leq \sup _{n} \frac{1}{n} \sum_{k=1}^{n} k^{-s}\left[M_{k}\left(q\left(\frac{\left|\Delta_{v}^{m} x_{k}\right|^{\frac{1}{k}}}{\rho}\right)\right)\right]^{p_{k}}<\infty$

From (3.61) and (3.62), it follows that $x \in \Lambda_{\mathcal{M}}\left(\Delta_{v}^{m}, q, s\right)$.Thus $\Lambda_{\mathcal{M}}\left(\Delta_{v}^{m}, p, q, s\right) \subset \Lambda_{\mathcal{M}}\left(\Delta_{v}^{m}, q, s\right)$.

(ii) Let $p_{k} \geq 1$ for each $\mathrm{k}$ and $\sup p_{k}<\infty$, and let $x \in \Lambda_{\mathcal{M}}\left(\Delta_{v}^{m}, q\right.$, s).Then

$$
\sup _{n} \frac{1}{n} \sum_{k=1}^{n} k^{-s}\left[M_{k}\left(q\left(\frac{\left|\Delta_{v}^{m} x_{k}\right| \frac{1}{k}}{\rho}\right)\right)\right]<\infty
$$

Since $1 \leq p_{k} \leq \operatorname{supp}_{k}<\infty$, we have 


$$
\begin{gathered}
\sup _{n} \frac{1}{n} \sum_{k=1}^{n} k^{-s}\left[M_{k}\left(q\left(\frac{\mid \Delta_{v}^{m} x_{k} \frac{1}{k}}{\rho}\right)\right)\right]^{p_{k}} \leq \sup _{n} \frac{1}{n} \sum_{k=1}^{n} k^{-s}\left[M_{k}\left(q\left(\frac{\left|\Delta_{v}^{m} x_{k}\right|^{\frac{1}{k}}}{\rho}\right)\right)\right] \\
\Rightarrow \sup _{n} \frac{1}{n} \sum_{k=1}^{n} k^{-s}\left[M_{k}\left(q\left(\frac{\left|\Delta_{v}^{m} x_{k}\right|^{\frac{1}{k}}}{\rho}\right)\right)\right]^{p_{k}} \rightarrow 0 \text { as } n \rightarrow \infty
\end{gathered}
$$

This implies that $x \in \Lambda_{\mathcal{M}}\left(\Delta_{v}^{m}, p, q, s\right)$. Therefore, $\Lambda_{\mathcal{M}}\left(\Delta_{v}^{m}, q, s\right) \subset \Lambda_{\mathcal{M}}\left(\Delta_{v}^{m}, p, q, s\right)$.

\section{Conclusion}

We conclude that the sequence space that we have introduced, namely

$$
\Lambda_{\mathcal{M}}\left(\Delta_{v}^{m}, p, q, s\right)=\left\{x \in \Lambda(X): \sup _{n_{n}} \frac{1}{n} \sum_{k=1}^{n} k^{-s}\left[M_{k}\left(q\left(\frac{\left|\Delta_{v}^{m} x_{k}\right|^{\frac{1}{k}}}{\rho}\right)\right)\right]^{p_{k}}<\infty \text {, for some } \rho>0\right\}
$$

is not only a linear space but is also solid. The space extends the results of Raj et al [3] and Abbas and Kamel [7]. It further open doors for the extension of similar types of result for other spaces defined by Musielak-Orlicz functions.

\section{Acknowledgement}

The authors are thankful to Prof. M. A. Babagana and Dr. H. R. Bakari for their supports during the preparation of this paper.

\section{References}

[1]. H. Kizmaz, On certain sequence spaces, Canadian Math. Bull., 24 (2), 1981, 169-176.

[2]. M. Et and R. Colak, On generalized difference sequence spaces, Soochow J. Math., 21 (4), 1995, $377-386$.

[3]. K. S. Raj, S. K. Sharma and A. Gupta, Entire sequence spaces defined by Musielak-Orlicz function, Int. J. Math. Sci. and Appl. 1 (2), 2011,953-960.

[4]. J. Lindenstrauss and L. Tzafriri, On Orlicz sequence spaces, Israel J. Math. IO, 1971, 379-390.

[5]. J. Musielak, Orlicz spaces and modular spaces, Lecture Notes in Mathematics, 1034.1983

[6]. I. J. Maddox, Elements of functional analysis, Marcel Dekker Inc., New York and Basel, 1981.

[7]. N. M. Abbas, and R. A. Kamel, Orlicz space of difference analytic sequences, Mathematical Theory and Modeling, 4(3), 2014, 5157. 